\title{
Oxidative stress in genetically obese rats. A possible model of premature ageing
}

\author{
N. M. De Castro ${ }^{1}$, I. Baeza ${ }^{1}$, C. Vida ${ }^{1}$, N. Úbeda ${ }^{2}$, R. Manso ${ }^{3}$ and M. De La Fuente ${ }^{1}$ \\ ${ }^{1}$ Department of Physiology, Faculty of Biology, Complutense University of Madrid, 28040 Madrid, Spain, ${ }^{2}$ Department of \\ Pharmaceutical and Food Sciences, Faculty of Pharmacy, San Pablo CEU University, 28668 Madrid, Spain and \\ ${ }^{3}$ Department of Molecular Biology, Faculty of Sciences, Autonomous University of Madrid, 28049 Madrid, Spain
}

Obesity and the metabolic syndrome are characterised by an increased oxidative stress, which results in an imbalance between the production of reactive oxygen species (ROS) and the levels of antioxidant defences, with an increase of ROS ${ }^{(1)}$. One of the most recent theories of ageing is the 'oxidative-inflammatory' theory, in which an age-related progressive dysregulation of immune responses, as a result of an increase of the leucocyte oxidative stress, is associated with a higher rate of ageing ${ }^{(2)}$. Therefore, the aim of the present work was to study several oxidative stress parameters in immune organs and liver (as a key metabolic organ), in a murine model of genetic obesity. Male rats of two different strains (Zucker and Wistar) were used. Zucker rats are known as 'fa/fa' (which carry the homozygotic mutation of the leptin receptor gene and develop the metabolic syndrome) and 'Lean' (which carry the heterozygotic mutation and do not exhibit the syndrome, and acts as control for the 'fa/fa' group). Wistar rats were included as a strain control group. All animals were fed standard diet (PANLAB A04). At 6 months of age all groups were sacrificed and homogenates from spleen, thymus and liver were obtained. The oxidative stress parameters analysed were: xanthine oxidase activity (XO), as an oxidant parameter, total antioxidant capacity (TAC), total gluthatione (GSH) and gluthatione reductase (GR) activity as antioxidant defence parameters. In general, Zucker 'Lean' and Wistar rats showed similar levels of antioxidant defences (except for TAC in spleen, where Wistar rats showed increased levels with respect to 'Lean' and 'fa/fa'). However, Zucker 'fa/fa' rats showed lower levels of antioxidant defences with respect to 'Lean' and Wistar rats. GSH and GR in spleen, and TAC in liver did not show any changes. XO activity was increased in 'fa/fa' rats with respect to 'Lean', except in thymus where it was increased with respect to Wistar. These results indicate that obese rats could suffer premature oxidative stress and therefore premature ageing.
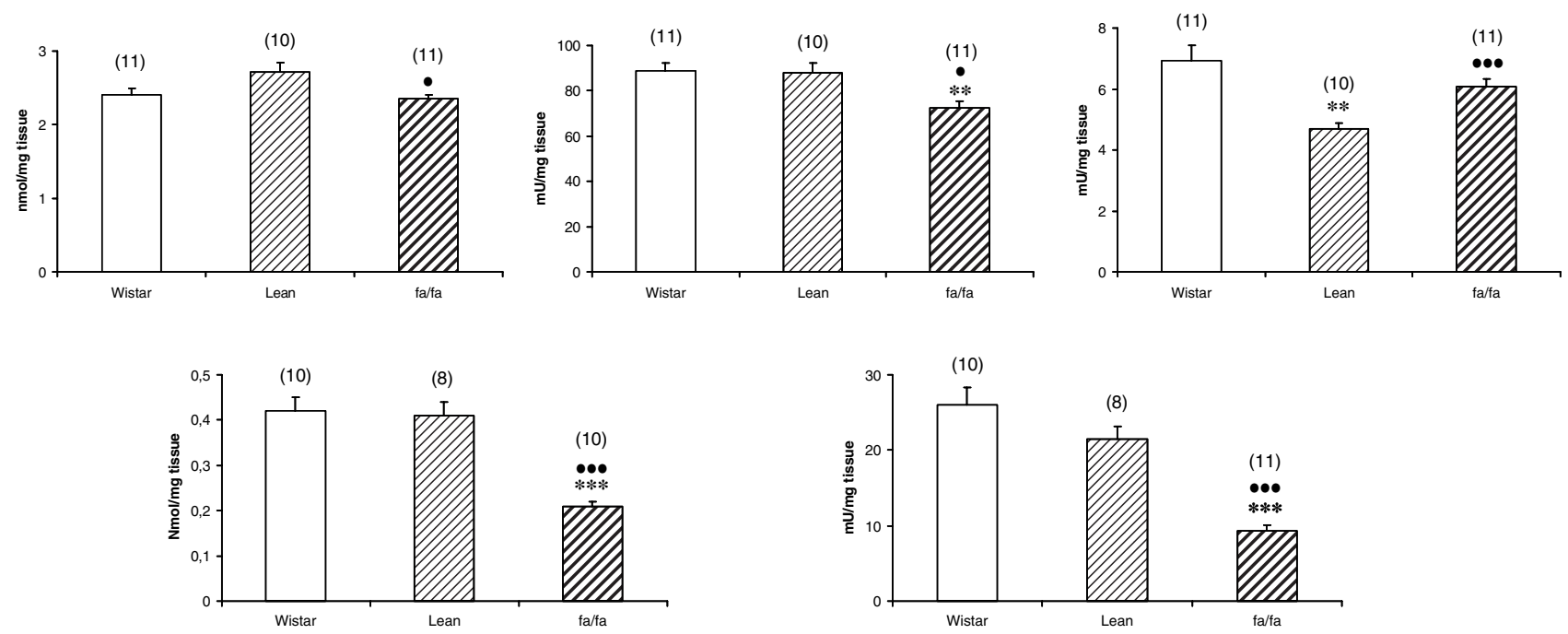

Data are expressed as means \pm SE of the number of animals used (in brackets).

$* * P<0.01 ; * * * P<0.001$ with respect to Wistar group. $\bullet P<0.05 ; \bullet \bullet P<0.001$ with respect to Lean group.

This work was financially support by MEC (DEP2006-56187-CO-02/PREV), UCM Research Group (910379ENEROINN) and RETICEF (RD06/0013/ 0003) (ISCIII) of Spain.

1. Roberts CK \& Sindhu KK (2009) Life Sci 84, 705-712.

2. De la Fuente M (2008) Neuroimmunomodulation 15, 213-223. 